\title{
Uniqueness of Gibbs fields with unbounded random interactions on unbounded degree graphs
}

\author{
Dorota Kępa-Maksymowicz ${ }^{1}$ Yuri Kozitsky ${ }^{1}$ (1)
}

Received: 31 October 2019 / Revised: 3 May 2020 / Accepted: 18 June 2020 / Published online: 29 June 2020

(c) The Author(s) 2020

\begin{abstract}
Gibbs fields with continuous spins are studied, the underlying graphs of which can be of unbounded vertex degree and the spin-spin pair interaction potentials are random and unbounded. A high-temperature uniqueness of such fields is proved to hold under the following conditions: (a) the vertex degree is of tempered growth, i.e., controlled in a certain way; (b) the interaction potentials $W_{x y}$ are such that $\left\|W_{x y}\right\|=\sup _{\sigma, \sigma^{\prime}}\left|W_{x y}\left(\sigma, \sigma^{\prime}\right)\right|$ are independent (for different edges $\langle x, y\rangle$ ), identically distributed and exponentially integrable random variables.
\end{abstract}

Keywords DLR equation - Specification · Quenched state · Unbounded disorder · High-temperature uniqueness · Animal

Mathematics Subject Classification 60K35 · 82B20

\section{Introduction and setup}

In this work, we continue studying quenched Gibbs fields with unbounded disorder [1] focusing on their high-temperature uniqueness. Permanent interest to this problem may arise from the fact that - even in the simplest case of an Ising model with unbounded random interactions - due to so-called Griffiths' singularities [2-4] at arbitrarily high temperatures there may exist arbitrarily large subsets of the underlying lattice, in which the spins are strongly correlated. Our work can be considered as a continuation of the research performed in [2,5-8]. Novel aspects here are: (a) instead of finite-valued spins we allow them to take values in arbitrary Polish spaces; (b) instead of regular underlying graphs (like $\mathbb{Z}^{d}$ ) we employ graphs of unbounded vertex degree, cf. $[9,10]$.

Supported in part by National Science Centre (NCN), Poland, Grant 2017/25/B/ST1/00051.

Yuri Kozitsky

jkozi@hektor.umcs.lublin.pl

1 Institute of Mathematics, Maria Curie-Sklodowska University, 20-031 Lublin, Poland 
Markov random fields on such underlying graphs [11] naturally appear in the following physical applications: (a) random (also quantum) fields on Riemannian manifolds, see e.g., [12]; (b) thermodynamic states of interacting oscillators based on networksso-called oscillating networks [13]; (c) thermodynamic states of continuous systems with spins, like ferrofluids, where (random) geometric graphs are used as underlying graphs $[14,15]$. We refer the interested reader to [10] for more details on this matter.

Let $\mathrm{G}=(\mathrm{V}, \mathrm{E})$ be a countably infinite graph with vertex and edge sets $\mathrm{V}$ and $\mathrm{E}$, respectively. We assume that $\mathrm{G}$ is connected, has no loops and multiple edges, and

$$
\forall x \in \mathrm{V} \quad n(x):=\#\{y \in \mathrm{V}: y \sim x\}<\infty,
$$

where $x \sim y$ denotes adjacency. In the latter case, we write the corresponding edge as $\langle x, y\rangle$. Let $S$ be a Polish space-a complete and separable metric space. By $\mathcal{B}(S)$ we denote the corresponding Borel $\sigma$-field, whereas $\mathcal{F}$ will stand for the smallest $\sigma$-field of subsets of $S^{\vee}$ such that the maps $S^{\vee} \ni \sigma \mapsto \sigma(x) \in S$ are measurable for all $x \in \mathrm{V}$. By $\mathcal{P}\left(S^{\vee}\right)$ we denote the set of all probability measures on $\left(S^{\vee}, \mathcal{F}\right)$. For a $\Delta \subset \mathrm{V}$, we write $\Delta^{c}=\mathrm{V} \backslash \Delta ; \Delta \Subset \mathrm{V}$ means $\Delta \subset \mathrm{V}$ and $\Delta$ is non-void and finite. For $\Delta \subset \mathrm{V}$, $\mathcal{F}_{\Delta}$ stands for the sub-field of $\mathcal{F}$ generated by the maps $S^{\vee} \ni \sigma \mapsto \sigma(x) \in S$ with $x \in \Delta$. For $\Delta \Subset \mathrm{V}$, a probability kernel $\pi_{\Delta}(\cdot \mid \cdot)$ is a function on $\left(\mathcal{F}, S^{\bigvee}\right)$ such that for any $\xi \in S^{\vee}, \pi_{\Delta}(\cdot \mid \xi)$ is in $\mathcal{P}\left(S^{\bigvee}\right)$, and for any $A \in \mathcal{F}, \pi_{\Delta}(A \mid \cdot)$ is $\mathcal{F}_{\Delta^{c}}$-measurable. Such a kernel is said to be proper if $\pi_{\Delta}(A \mid \cdot)=\mathbb{I}_{A}(\cdot)$ for any $A \in \mathcal{F}_{\Delta^{c}}$, cf. [16, page 14]. Here $\mathbb{I}_{A}(\xi)=1$ if $\xi \in A$, and $\mathbb{I}_{A}(\xi)=0$ otherwise. Let $\left\{\pi_{\Delta}\right\}_{\Delta \Subset \mathrm{V}}$ be the family of probability kernels such that for a given $\mu \in \mathcal{P}\left(S^{\vee}\right)$, one has

$$
\mu\left(A \mid \mathcal{F}_{\Delta^{c}}\right)=\pi_{\Delta}(A \mid \cdot),
$$

which holds $\mu$-almost surely for all $A \in \mathcal{F}$ and $\Delta \Subset \mathrm{V}$. Then one says [16, page 16] that $\mu$ is specified by $\left\{\pi_{\Delta}\right\}_{\Delta \Subset \mathrm{V}}$. In this case, all the kernels $\pi_{\Delta}$ are $\mu$-almost surely proper, and their family is $\mu$-almost surely consistent. The latter means that for $\mu$-almost all $\xi$ and all $A \in \mathcal{F}$, one has

$$
\int_{S^{\vee}} \pi_{\Lambda}(A \mid \eta) \pi_{\Delta}(d \eta \mid \xi)=\pi_{\Delta}(A \mid \xi),
$$

which holds for any pair of subsets such that $\Lambda \subset \Delta$. It should be pointed out that (1.2) holds if and only if

$$
\int_{S^{\vee}} \pi_{\Delta}(A \mid \xi) \mu(d \xi)=\mu(A),
$$

for all $A \in \mathcal{F}$ and $\Delta \Subset \mathrm{V}$. The condition (1.3) can be considered as the equation which defines the random fields specified by the family of kernels $\left\{\pi_{\Delta}\right\}_{\Delta \Subset V}$. It is known under the name Dobrushin-Lanford-Ruelle (DLR) equation. A paramount problem of the theory is then the number of such fields specified by a given family $\left\{\pi_{\Delta}\right\}_{\Delta \Subset V}$, see [16].

Let $W=\left\{W_{x y}\right\}_{\langle x, y\rangle \in \mathrm{E}}$ be a family of symmetric measurable functions $W_{x y}$ : $S \times S \rightarrow \mathbb{R}$, interpreted as the interaction potentials, i.e., $W_{x y}(\sigma(x), \sigma(y)) \in \mathbb{R}$ is 
the energy related to the spin values $\sigma(x), \sigma(y) \in S$. For $\Delta \Subset \mathrm{V}$ and $\xi \in S^{\mathrm{V}}$, we set

$$
H_{\Delta}(\sigma \mid \xi)=-\frac{1}{2} \sum_{x, y \in \Delta, x \sim y} W_{x y}(\sigma(x), \sigma(y))-\sum_{x \in \Delta, \Delta_{y \in \Delta^{c}, x \sim y}} W_{x y}(\sigma(x), \xi(y)) .
$$

Let $\chi=\left\{\chi_{x}\right\}_{x \in \mathrm{V}}$ be a family of $\chi_{x} \in \mathcal{P}(S)$. For $\Delta \subset \mathrm{V}$, by $\chi_{\Delta}$ we denote the corresponding product measure on $S^{\Delta}$. The elements of the latter set are denoted by $\sigma_{\Delta}$. The local Gibbs specification corresponding to (1.4) and $\chi$ is the collection of the following probability kernels

$$
\begin{aligned}
\pi_{\Delta}^{\beta}(A \mid \xi) & =\frac{1}{Z_{\Delta}^{\beta}(\xi)} \int_{S^{\Delta}} \mathbb{I}_{A}\left(\sigma_{\Delta} \times \xi_{\Delta^{c}}\right) \\
& \times \exp \left[-\beta H_{\Delta}(\sigma \mid \xi)\right] \chi_{\Delta}\left(d \sigma_{\Delta}\right), \quad A \in \mathcal{F}, \quad \beta>0 .
\end{aligned}
$$

Here $\beta$ is the inverse absolute temperature, $Z_{\Delta}^{\beta}(\xi)$ is the normalizing factor-partition function, and the juxtaposition stands for the element of $S^{\vee}$ such that

$$
\left(\sigma_{\Delta} \times \xi_{\Delta^{c}}\right)(x)=\sigma(x), \text { for } x \in \Delta ; \quad\left(\sigma_{\Delta} \times \xi_{\Delta^{c}}\right)(x)=\xi(x), \text { for } x \in \Delta^{c} .
$$

Then by $\mathcal{G}^{\beta}(W, \chi)$ we denote the set of $\mu \in \mathcal{P}\left(S^{\vee}\right)$ that solve the DLR equation (1.3) with the specification defined in (1.5).

As mentioned above, we study the case where the interaction potentials $W_{x y}$ are random functions, and hence $\mathcal{G}^{\beta}(W, \chi)$ is a random set, see [1] for a more detailed presentation of the corresponding notions. In the Ising model studied in [2,7], $W_{x y}(\sigma(x), \sigma(y))=J_{x y} \sigma(x) \sigma(y)$ with random $J_{x y}$. The aim of this work is to find a sufficient condition under which $\mathcal{G}^{\beta}(W, \chi)$ is almost surely a singleton.

\section{Tempered graphs and the statement}

We begin by introducing the underlying graph $\mathrm{G}$ and the corresponding graph-related terminology and facts. Afterward, we formulate and comment our result.

\subsection{Tempered graphs}

As mentioned above, the underlying graph $G=(V, E)$ is supposed to satisfy (1.1), which means that is locally finite. At the same time, we allow $\mathrm{G}$ to be such that

$$
\sup _{x \in \mathrm{V}} n(x)=+\infty
$$

Let $\mathrm{G}^{\prime}=\left(\mathrm{V}^{\prime}, \mathrm{E}^{\prime}\right)$ be a subgraph of $\mathrm{G}$, i.e., $\mathrm{G}^{\prime}$ is a graph and $\mathrm{V}^{\prime} \subset \mathrm{V}, \mathrm{E}^{\prime} \subset \mathrm{E}$. In this case, we write $G^{\prime} \subset \mathrm{G}$. A finite connected subgraph of $\mathrm{G}$ is called an animal. Such zoo-terminology is used in the theory of Gibbs states based on cluster expansions, see, e.g., [17], and in percolation-related works, e.g., [18]. For an animal A, by V(A) and 
$E(A)$ we denote its vertex and edge sets, respectively. For graphs of bounded vertex degree, i.e., those satisfying

$$
\sup _{x \in \mathrm{V}} n(x)=: \bar{n}<+\infty
$$

one has

$$
G(\mathrm{~A} ; g):=\frac{1}{|\mathrm{~V}(\mathrm{~A})|} \sum_{x \in \mathrm{V}(\mathrm{A})} g(n(x)) \leq g(\bar{n}),
$$

holding for each unbounded $g:[1,+\infty) \rightarrow[0,+\infty)$. Then a possible way of controlling deviations from the boundedness as in (2.2) is to impose weaker versions of (2.3), which would make impossible accumulations of hubs-vertices of high degree. This idea was realized in our previous work [9] the results of which will be used here. Since we are going to modify the basic notion of [9]—and also for reader's convenience-we begin by presenting facts and notions related to this matter.

A path in $\mathrm{G}$ is a finite sequence of its vertices $\vartheta=\left\{x_{l}: l=0,1, \ldots, n\right\}$ such that $x_{l} \sim x_{l+1}$ for all $l$. Vertices $x_{0}$ and $x_{n}$ are its origin and terminus, respectively, and $n=:\|\vartheta\|$ is the length of the path. It is equal to the number of consecutive pairs $x_{l}, x_{l+1}$ in $\vartheta$. By writing $\vartheta(x, y)$ we indicate that the path originates at $x$ and terminates at $y$, and thereby connects these vertices. For consecutive $x_{l}, x_{l+1}$, we say that the path leaves $x_{l}$ and enters $x_{l+1}$. Some of the vertices can appear in $\vartheta$ several times. By $v_{\vartheta}(x), x \in \mathrm{V}$, we denote the number of times $\vartheta$ leaves $x$, including $v_{\vartheta}(x)=0$ if $x$ is not in $\vartheta$. A subsequence $\vartheta^{\prime}$ of $\vartheta$ is a subpath of the latter if $\vartheta^{\prime}$ is a path on its own. A path $\vartheta$ is called simple if $v_{\vartheta}(x) \leq 1$ for all $x$. A simple path with coinciding origin and terminus is called a cycle. In a simple path, each vertex appears only once-except, possibly, for the origin and terminus which can coincide if $\vartheta$ is a cycle. Each path $\vartheta(x, y)$ contains a subpath $\vartheta^{\prime}(x, y)$, which is simple.

For a path $\vartheta$, let $\mathrm{G}_{\vartheta}=\left(\mathrm{V}_{\vartheta}, \mathrm{E}_{\vartheta}\right)$ be its graph. Here $\mathrm{V}_{\vartheta}$ and $\mathrm{E}_{\vartheta}$ consist of the vertices $x_{l}$ and edges $\left\langle x_{l}, x_{l+1}\right\rangle$, respectively, where each of them is counted only once. Clearly, $\mathrm{G}_{\vartheta}=\left(\mathrm{V}_{\vartheta}, \mathrm{E}_{\vartheta}\right)$ is an animal since the connectivity is defined as the existence of a path connecting each pair of distinct vertices. By Euler's classical theorem, see, e.g., [19, page 51] it is possible to show that each $A$ is in fact $\mathrm{G}_{\vartheta}$ for a certain path $\vartheta$ such that $v_{\vartheta}(x) \leq n(x)$, see the proof of Lemma 17 in [9] for more detail. For $x, y \in \mathrm{V}$, the distance between them is defined as

$$
\rho(x, y)=\min \|\vartheta(x, y)\|,
$$

where min is taken over all paths connecting $x$ and $y$. With this distance $\mathrm{G}$ is a metric space.

Now let $G$ be such that (2.1) holds, and $g$ be an increasing ad infinitum function. Then $G(\mathrm{~A} ; g)$ defined in (2.3) can be made arbitrarily big just by picking a hub and then including in $A$ this hub and a couple of its neighbors. Loosely speaking, a graph is declared tempered if hubs are sparse, i.e., most of them are at large distances (2.4) of each other. This means that big animals can contain only few hubs, and hence $G(\mathrm{~A} ; g)$ for such animals can be bounded, uniformly for all big A. Let us make this idea more precise. For given $x \in \mathrm{V}$ and $r \in \mathbb{N}$, let $\mathrm{G}_{r}(x)$ be the subgraph with vertex 
set $\mathrm{V}_{r}(x)=\{y \in \mathrm{V}: \rho(x, y) \leq r\}$ and edge set consisting of all those edges of $\mathrm{G}$ both endpoints of which are in $\mathrm{V}_{r}(x)$. Now we set

$$
\mathcal{A}_{r}(x)=\left\{\mathrm{A} \subset \mathrm{G}_{r}(x):|\mathrm{V}(\mathrm{A})| \geq r+1\right\}
$$

In the definition below, by $g$ we mean any increasing ad infinitum function $g:[1+$ $\infty) \rightarrow[0,+\infty)$.

Definition $1 \mathrm{G}$ is said to be $g$-tempered if for every $x \in \mathrm{V}$, there exists a strictly increasing sequence $\left\{N_{k}\right\}_{k \in \mathbb{N}} \subset \mathbb{N}$ such that

$$
\sup _{x \in \mathrm{V}} \sup _{k \in \mathbb{N}}\left(\max _{\mathrm{A} \in \mathcal{A}_{N_{k}}(x)} G(\mathrm{~A} ; g)\right)=: \gamma<\infty .
$$

The following rooted tree, cf. [8, Section 4], can serve as a natural example characterizing the property just defined. In this tree, the root $x$ has two neighbors, which have three further neighbors each (not counting $x$ ). These neighbors have four further neighbors each, and so on. It is an easy exercise to show that such a graph is not $g$-tempered for any unbounded $g$. Notably, in this graph each hub has an even bigger hub at distance one.

Another natural example characterizing the property defined above is provided by so-called repulsive graphs, see [9]. Some of such graphs were introduced in [5, Theorem 3]. To describe them we set $m_{-}(x, y)=\min \{n(x) ; n(y)\}, x, y \in \mathrm{V}$, and let $\phi:[1,+\infty) \rightarrow[0,+\infty)$ be an increasing ad infinitum function. Then the set of graphs $\mathbb{G}_{-}(\phi)$ is defined by the following property: for each $G \in \mathbb{G}_{-}(\phi)$, there exists $n_{*} \in \mathbb{N}$ such that, for each $x, y \in \mathrm{V}$, the following holds

$$
\rho(x, y) \geq \phi\left(m_{-}(x, y)\right)
$$

whenever $m_{-}(x, y) \geq n_{*}$.

Proposition 1 Assume that there exists a strictly increasing sequence $\left\{t_{k}\right\}_{k \in \mathbb{N}}, t_{k} \rightarrow$ $+\infty$, such that $g$ and $\phi$ satisfy

$$
\sum_{k=1}^{\infty} \frac{g\left(t_{k+1}\right)}{\phi\left(t_{k}\right)}<+\infty
$$

Then each $G \in \mathbb{G}_{-}(\phi)$ is g-tempered.

This statement resembles Theorem 5 of [9]. We give its proof in this article below since the definition of temperedness used here is a bit different from that made in [9, Definition 1]. To this end we will need some facts proved in [9].

Proposition 2 [9, Lemma 17] Let $G$ be an arbitrary graph. Given $\lambda>1$ and an animal $A \subset G$, let $B \subset V(A)$ be such that $\rho(x, y) \geq \lambda$ for each pair of distinct $x, y \in B$. Then

$$
|B| \leq \max \left\{1 ; \frac{2|V(A)|-1}{\lambda}\right\} \text {. }
$$


Before formulating the next result we recall that $\phi$ is an increasing ad infinitum function and the ball $\mathrm{V}_{r}(x)=\{y: \rho(x, y) \leq r\}$ is the set of vertices of $\mathrm{G}_{r}(x)$, see (2.5).

Proposition 3 [9, Lemma 18] Let $G$ be in $\mathbb{G}_{-}(\phi)$. Then for each $x \in V$, there exists a strictly increasing sequence $\left\{N_{k}\right\}_{k \in \mathbb{N}} \subset \mathbb{N}$ such that, for each $k \in \mathbb{N}$, the following holds

$$
\max _{y \in V_{N_{k}}(x)} n(y) \leq \phi^{-1}\left(2 N_{k}+1\right) .
$$

Proof of Proposition 1 For a given $x \in \mathrm{V}$, let $\left\{N_{k}\right\}_{k \in \mathbb{N}}$ be as in Proposition 3. For $\mathrm{A} \in$ $\mathcal{A}_{N_{k}}(x)$, see (2.5), by (2.10) we then have $\phi\left(n_{\mathrm{A}}\right) \leq 2|\mathrm{~V}(\mathrm{~A})|, n_{\mathrm{A}}:=\max _{y \in \mathrm{V}(\mathrm{A})} n(y)$. Now let $\left\{t_{k}\right\}_{k \in \mathbb{N}}$ be such that (2.8) holds. Set $\mathrm{M}_{k}=\left\{y \in \mathrm{V}(\mathrm{A}): n(y) \in\left[t_{k}, t_{k+1}\right)\right\}$, $k \in \mathbb{N}$. By (2.7) it follows that $\rho\left(y, y^{\prime}\right) \geq \phi\left(t_{k}\right)$ whenever $y, y^{\prime} \in \mathrm{M}_{k}$. By (2.9) this yields

$$
\left|\mathrm{M}_{k}\right| \leq \frac{2|\mathrm{~V}(\mathrm{~A})|}{\phi\left(t_{k}\right)}
$$

With the help of this estimate we then have

$$
\sum_{y \in \mathrm{V}(\mathrm{A})} g(n(y)) \leq \sum_{k=1}^{\infty} g\left(t_{k+1}\right)\left|\mathrm{M}_{k}\right| \leq 2\left(\sum_{k=1}^{\infty} \frac{g\left(t_{k+1}\right)}{\phi\left(t_{k}\right)}\right)|\mathrm{V}(\mathrm{A})|=: \gamma|\mathrm{V}(\mathrm{A})|,
$$

which completes the proof.

Now let $\Sigma_{r}(x)$ be the set of all simple paths $\vartheta$ (excluding cycles) such that $\mathrm{G}_{\vartheta} \subset$ $\mathrm{G}_{r}(x)$ and $\|\vartheta\| \geq r$; hence, $\left|\mathrm{V}_{\vartheta}\right| \geq r+1$. Clearly, $\mathrm{G}_{\vartheta} \in \mathcal{A}_{r}(x)$ for each $\vartheta \in \Sigma_{r}(x)$, see (2.5). Set $g_{1}(t)=\log t, g_{2}(t)=t \log t, t \geq 1$, and also $\Sigma_{r}^{N}(x)=\left\{\vartheta \in \Sigma_{r}(x)\right.$ : $\|\vartheta\|=N\}, \mathcal{A}_{r}^{N}(x)=\left\{\mathrm{A} \in \mathcal{A}_{r}(x):|\mathrm{V}(\mathrm{A})|=N\right\}$. The next statement provides estimates of the cardinalities of these sets.

Proposition 4 Let $G$ be $g_{1}$-tempered, $\gamma>0$ be as in (2.6), $x \in V$ and $\left\{N_{k}\right\}_{k \in \mathbb{N}}$ be as in Definition 1. Then for each $k \in \mathbb{N}$ and $N \geq N_{k}$, the following holds $\left|\Sigma_{N_{k}}^{N}(x)\right| \leq$ $\exp (\gamma N)$. If $G$ is $g_{2}$-tempered, then $\left|\mathcal{A}_{r}^{N}(x)\right| \leq \exp (\gamma N)$ holding for all $N \geq N_{k}+1$.

Proof Let $\Theta$ be a finite family of paths in a graph G. Proceeding as in the proof of [9, Lemma 15] we obtain the following estimate of its cardinality

$$
|\Theta| \leq \max _{\vartheta \in \Theta} \exp \left(\sum_{y \in \vartheta} \log n(y)\right)=\max _{\vartheta \in \Theta} \exp \left(\sum_{y \in \bigvee_{\vartheta}} v_{\vartheta}(y) \log n(y)\right),
$$

where $v_{\vartheta}(y)$ is the number of times $\vartheta$ leaves $y$. For $\vartheta \in \Sigma_{N_{k}}^{N}(x)$, we have $v_{\vartheta}(y) \leq 1$, which by (2.11) and (2.6) yields the estimate in question. As mentioned above, for each $\mathrm{A}$, there exists $\vartheta$ such that $\mathrm{A}=\mathrm{G}_{\vartheta}$ and $v_{\vartheta}(y) \leq n(y)$ for each $y \in \mathrm{V}_{\vartheta}=\mathrm{V}(\mathrm{A})$. Let $\Theta_{r}^{N}(x)$ be the family of all paths in $\mathrm{G}_{r}(x)$ such that $\left|\mathrm{G}_{\vartheta}\right|=N$ and $v_{\vartheta}(y) \leq n(y)$ for all $y \in \mathrm{V}_{\vartheta}$. Then $\left|\mathcal{A}_{N_{k}}^{N}(x)\right| \leq\left|\Theta_{N_{k}}^{N}(x)\right| \leq \exp (\gamma N)$, where the latter estimate is obtained in the same way as the estimate for $\left|\Sigma_{N_{k}}^{N}(x)\right|$. This completes the proof. 
Now let us make some comments.

(i) As mentioned above, in graphs with unbounded degrees $G(\mathrm{~A} ; g)$ can be arbitrarily big if one takes A 'small' and containing a hub. According to Definition 1, in a tempered graph there exists a scale of 'big enough' animals, for which $G(\mathrm{~A} ; g) \leq \gamma$ with a universal (for this $\mathrm{G}$ ) constant $\gamma$. This scale is defined by means of ballstypical objects for metric spaces. Temperedness introduced in this work is a bit stronger than the corresponding notion established in [9, Definition 1]. The main difference is that in the present case, the condition $G(\mathrm{~A} ; g) \leq \gamma$ is satisfied by all animals $\mathrm{A} \subset \mathrm{G}_{N_{k}}(x)$-not only those for which $x \in \mathrm{V}(\mathrm{A})$ and $|\mathrm{V}(\mathrm{A})|=N_{k}$, as is assumed in [9].

(ii) Proposition 1 provides a basic example of a tempered graph. It is, however, not the only one satisfying Definition 1 . For $\mathrm{G} \in \mathbb{G}_{-}(\phi)$, if $n(x) \geq n_{*}$, then the ball $\mathrm{B}_{x}:=\{y: \rho(x, y) \leq \phi(n(x))-1\}$ contains no $z \in \mathrm{V}$ such that $n(z) \geq n(x)$. It is clear that the graph remains tempered if one allows such balls contain up to a fixed number of hubs $z$ for which $n(z)=n(x)$. In other words, our tempered graphs include repulsive graphs as a particular case.

(iii) In [10, Assumption 2.1], there was used a condition based on the weighted summability of

$$
m_{\theta}(x)=\sum_{y: y \sim x}[n(x) n(y)]^{\theta}, \quad \theta>0 .
$$

In mathematical chemistry, $m_{\theta}$ is known as generalized Randić index, see [20]. It turns out, see [10, Theorem 5.2], that graphs from $\mathbb{G}_{+}(\phi)$ satisfy this condition for a specific choice of $\phi$. Here the family $\mathbb{G}_{+}(\phi)$ is defined by the repulsive condition as in (2.7) with $m_{-}$replaced by $m_{+}(x, y)=\max \{n(x) ; n(y)\}$. More on the properties of $\mathbb{G}_{+}(\phi)$ and Randić indices in this context can be found in [9].

\subsection{The statement}

Let $\mathcal{C}$ be the Banach space of symmetric bounded continuous functions $S \times S \ni$ $\left(s, s^{\prime}\right) \mapsto f\left(s, s^{\prime}\right) \in \mathbb{R}$, equipped with the norm

$$
\|f\|=\sup _{\left(s, s^{\prime}\right) \in S \times S}\left|f\left(s, s^{\prime}\right)\right|
$$

and with the corresponding Borel $\sigma$-field. We then equip $\mathcal{C}^{\mathrm{E}}$ with the product topology and the product $\sigma$-field of subsets. Let $(\Omega, \mathcal{O}, P)$ be a probability space. Then a random interaction potential is a measurable map $W: \Omega \rightarrow \mathcal{C}^{\mathrm{E}}$. In this work, it is supposed to satisfy:

(i) $\forall\langle x, y\rangle \in \mathrm{E} \quad\left\|W_{x y}\right\|=\sup _{\sigma(x), \sigma(y) \in S}\left|W_{x y}(\sigma(x), \sigma(y))\right|<\infty$,

(ii) $\left\{\left\|W_{x y}\right\|,\langle x, y\rangle \in \mathrm{E}\right\}$ are i.i.d.,

(iii) $\forall\langle x, y\rangle \in \mathrm{E} \quad \forall \beta>0 \quad \mathbb{E}\left[\exp \left(\beta\left\|W_{x y}\right\|\right)\right]<\infty$. 
By (1.1) and property (i) above the interaction potential is almost surely absolutely summable, cf. [16, Subsec. 2.11, pages 28, 29]. Then, for all $\beta>0$, by [16, Theorem 4.23 claim (a), page 72$], \mathcal{G}^{\beta}(W, \chi)$ is almost surely non-void. The result of this paper is the following statement.

Theorem 1 Let $G$ be g-tempered with $g(t)=\log t$, cf. Proposition 4, and the conditions in (2.12) be satisfied. Then there exist $\beta_{*}>0$ such that, for all $\beta<\beta_{*}$, the set $\mathcal{G}^{\beta}(W, \chi)$ is almost surely a singleton.

Let us make some comments to this statement.

(a) Up to the best of our knowledge, this is the first result of this kind for unbounded degree graphs. The temperedness of the underlying graph as in Definition 1 is crucial for the uniqueness of Gibbs fields constructed thereon-even in the simplest case of nonrandom interactions of spins taking values \pm 1 . The Ising model on the rooted tree mentioned above has multiple phases at all temperatures, see [8, Section 4].

(b) Perhaps, the first rigorous result concerning uniqueness of Gibbs fields with regular underlying graphs (in fact, lattices), finite-valued spins and unbounded random interactions was obtained in [5]. Despite the title of that paper, the technique used there- 'gluing out' vertices of the original lattice with subsequent passing to coarse-grained graphs of a special structure-was quite complicated and not everywhere correct. For example, the proof of Proposition 3 was based on a wrong estimate of the distance between vertices of the coarse-grained graph. Fortunately, this gap can be bridged and thus the main statement of [5] survives. Our resultessentially more general-is obtained in a much more natural and transparent way.

(c) Assumption (i) of (2.12) is crucial. Our result does not cover the case

$$
W_{x y}(\sigma(x), \sigma(y))=J_{x y} \sigma(x) \sigma(y), \quad \sigma(x), \sigma(y) \in \mathbb{R},
$$

with random $J_{x y}$. For such models, only existence of Gibbs fields on graphs satisfying (2.2) has been established so far, see [1].

(d) The i.i.d. condition in assumption (ii) of (2.12) is typical for such situations [2,5-7].

\section{The proof}

The proof will be based on the estimates obtained in Proposition 4 and Lemma 1, see below, proved in the spirit of similar statements in [2,21]. For $\langle x, y\rangle \in \mathrm{E}$ and $\beta>0$, set

$$
\varkappa_{x y}(\beta)=\exp \left(4 \beta\left\|W_{x y}\right\|\right)-1 .
$$

For $\Delta \Subset \mathrm{V}$, by $\partial_{\mathrm{V}}^{+} \Delta$ and $\partial_{\mathrm{V}}^{-} \Delta$ we denote the outer and inner vertex boundaries of $\Delta$, respectively. That is,

$$
\begin{aligned}
& \partial_{\mathrm{V}}^{+} \Delta=\left\{y \in \Delta^{c}: \exists x \in \Delta \quad\langle x, y\rangle \in \mathrm{E}\right\} \\
& \partial_{\mathrm{V}}^{-} \Delta=\left\{x \in \Delta: \exists y \in \Delta^{c} \quad\langle x, y\rangle \in \mathrm{E}\right\} .
\end{aligned}
$$


For $z \in \Delta \backslash \partial_{\mathrm{V}}^{-} \Delta$ and $x \in \partial_{\mathrm{V}}^{-} \Delta$, define

$$
Q_{\Delta}^{\beta}(z, x)=\sum_{\vartheta} \prod_{\langle u, v\rangle \in \mathrm{E}_{\vartheta}} \varkappa_{u v}(\beta)
$$

where the sum is taken over all simple paths in $\Delta$ whose origin and terminus are $z$ and $x$, respectively. Note that, for fixed $\beta, \Delta$ and $z, x, Q_{\Delta}^{\beta}(x, y)$ is a random variable, the expected value of which can be estimated by employing item (iii) of (2.12).

Along with the measures (1.5), we consider also local measures on $\mathcal{P}\left(S^{\Delta}\right)$ of the following form

$$
v_{\Delta}^{\beta}\left(d \sigma_{\Delta} \mid \xi\right)=\frac{1}{\widetilde{Z}_{\Delta}^{\beta}(\xi)} \exp \left[-\beta \widetilde{H}_{\Delta}\left(\sigma_{\Delta} \mid \xi\right)\right] \chi_{\Delta}\left(d \sigma_{\Delta}\right)
$$

where

$$
\begin{aligned}
\widetilde{H}_{\Delta}\left(\sigma_{\Delta} \mid \xi\right) & =-\frac{1}{2} \sum_{x, y \in \Delta, x \sim y}\left(W_{x y}(\sigma(x), \sigma(y))+\left\|W_{x y}\right\|\right) \\
& -\sum_{x \in \Delta, y \in \partial_{V}^{+} \Delta, x \sim y} W_{x y}(\sigma(x), \xi(y)),
\end{aligned}
$$

and $\widetilde{Z}_{\Delta}^{\beta}(\xi)$ is the corresponding normalization factor. Since $H$ defined in (1.4) and $\widetilde{H}$ differ only on an additive term independent of $\sigma$, for each $\mathcal{F}_{\Delta}$-measurable $f: S^{\vee} \rightarrow \mathbb{R}$, it follows that

$$
\int_{S^{V}} f(\sigma) \pi_{\Delta}^{\beta}(d \sigma \mid \xi)=\int_{S^{\Delta}} h\left(\sigma_{\Delta}\right) \nu_{\Delta}^{\beta}\left(d \sigma_{\Delta} \mid \xi\right)
$$

where $h: S^{\Delta} \rightarrow \mathbb{R}$ is such that $f(\sigma)=h\left(\sigma_{\Delta}\right)$ for all $\sigma \in S^{\vee}$. Given $z \in \mathrm{V}$ and a continuous $h: S \rightarrow[0,1]$, we denote

$$
M_{\Delta, z}^{\beta}(h \mid \xi)=\int_{S^{\Delta}} h(\sigma(z)) \nu_{\Delta}^{\beta}\left(d \sigma_{\Delta} \mid \xi\right)
$$

Lemma 1 For each $\Delta \Subset V, z \in \Delta \backslash \partial_{V}^{-} \Delta$ and arbitrary $\xi, \eta \in S^{V}$, (with probability one) the following holds

$$
\left|M_{\Delta, z}^{\beta}(h \mid \xi)-M_{\Delta, z}^{\beta}(h \mid \eta)\right| \leq \sum_{x \in \partial_{V}^{-} \Delta} Q_{\Delta}^{\beta}(z, x) .
$$


Proof Since both sides of (3.5) are random, we are going to prove this inequality pointwise in $\omega \in A$ with $P(A)=1$. By (3.3) and (3.4) we obtain

$$
\begin{aligned}
M_{\Delta, z}^{\beta}(h \mid \xi)- & M_{\Delta, z}^{\beta}(h \mid \eta) \\
= & \int_{S^{\Delta}} \int_{S^{\Delta}}(h(\sigma(z))-h(\tilde{\sigma}(z))) v_{\Delta}^{\beta}\left(d \sigma_{\Delta} \mid \xi\right) v_{\Delta}^{\beta}\left(d \tilde{\sigma}_{\Delta} \mid \eta\right) \\
= & \frac{1}{\widetilde{Z}_{\Delta}^{\beta}(\xi) \widetilde{Z}_{\Delta}^{\beta}(\eta)} \int_{S^{\Delta}} \int_{S^{\Delta}}(h(\sigma(z))-h(\tilde{\sigma}(z))) \\
& \times \prod_{\langle x, y\rangle \in \mathrm{E}_{\Delta}}\left(1+\Gamma_{x y}\right) \Psi_{\Delta}(\xi, \eta) \chi_{\Delta}\left(d \sigma_{\Delta}\right) \chi_{\Delta}\left(d \tilde{\sigma}_{\Delta}\right) \\
= & \frac{1}{\widetilde{Z}_{\Delta}^{\beta}(\xi) \widetilde{Z}_{\Delta}^{\beta}(\eta)} \sum_{\mathrm{E}^{\prime} \subset \mathrm{E}_{\Delta}} \int_{S^{\Delta}} \int_{S^{\Delta}}(h(\sigma(z))-h(\tilde{\sigma}(z))) \\
& \times \Gamma\left(\mathrm{E}^{\prime}\right) \Psi_{\Delta}(\xi, \eta) \chi_{\Delta}\left(d \sigma_{\Delta}\right) \chi_{\Delta}\left(d \tilde{\sigma}_{\Delta}\right),
\end{aligned}
$$

where

$$
\begin{aligned}
\Gamma\left(\mathrm{E}^{\prime}\right)= & \prod_{\langle x, y\rangle \in \mathrm{E}^{\prime}} \Gamma_{x y}, \\
\Gamma_{x y}= & \exp \left(\beta\left[W_{x y}(\sigma(x), \sigma(y))+\left\|W_{x y}\right\|\right]\right. \\
& \left.+\beta\left[W_{x y}(\tilde{\sigma}(x), \tilde{\sigma}(y))+\left\|W_{x y}\right\|\right]\right)-1, \\
\Psi_{\Delta}(\xi, \eta)= & \prod_{x \in \Delta, y \in \partial_{\mathrm{V}}^{+} \Delta, x \sim y} \exp \left[\beta W_{x y}(\sigma(x), \xi(y))+\beta W_{x y}(\tilde{\sigma}(x), \eta(y))\right] .
\end{aligned}
$$

One observes that each $\Gamma_{x y} \geq 0$. To get this property of $\Gamma_{x y}$ we added $\left\|W_{x y}\right\|$ to the corresponding interaction terms. Fix some $\mathrm{E}^{\prime}$ in the last line in (3.6) and consider the subgraph $\mathrm{G}^{\prime}=\left(\Delta, \mathrm{E}^{\prime}\right)$. If $z \in \Delta \backslash \partial_{\mathrm{V}}^{-} \Delta$ and the boundary $\partial_{\mathrm{V}}^{-} \Delta$ lie in distinct connected components of $\mathrm{G}^{\prime}$, then the integrals over the spins $\sigma(z), \tilde{\sigma}(z)$ and over $\sigma(x), \tilde{\sigma}(x)$ with $x \in \partial_{\mathrm{V}}^{-} \Delta$ get independent and hence the left-hand side of (3.6) vanishes as the term $h(\sigma(x))-h(\tilde{\sigma}(x))$ is antisymmetric with respect to the interchange $\sigma \leftrightarrow \tilde{\sigma}$, whereas all $\Gamma_{x y}$ are symmetric and the only break of this symmetry is related to the fixed boundary spins $\xi(y)$ and $\eta(y), y \in \partial_{\mathrm{V}}^{+} \Delta$. Therefore, each non-vanishing term in (3.6) corresponds to a path $\vartheta(z, x)$ connecting $z$ to some $x \in \partial_{\mathrm{V}}^{-} \Delta$. Each such a path $\vartheta(z, x)$ can be taken simple as every path contains a simple subpath with the same origin and terminus. Let $\Theta_{\Delta}(z)$ be the family of all simple paths in $\Delta$ connecting $z$ and some $x \in \partial_{\mathrm{V}}^{-} \Delta$. Then the sum in (3.6) can be restricted to those $\mathrm{E}^{\prime} \subset \mathrm{E}_{\Delta}$ that contain the edges of at least one $\vartheta \in \Theta_{\Delta}(z)$. For such a path $\vartheta$, set $\mathcal{E}_{\vartheta}=\left\{\mathrm{E}^{\prime} \subset \mathrm{E}_{\Delta}: \mathrm{E}_{\vartheta} \subset \mathrm{E}^{\prime}\right\}$. Note that, for distinct $\vartheta, \vartheta^{\prime} \in \Theta_{\Delta}(z)$, the corresponding families $\mathcal{E}_{\vartheta}$ and $\mathcal{E}_{\vartheta^{\prime}}$ need not 
be disjoint as they may include those $\mathrm{E}^{\prime}$ which contain both $\mathrm{E}_{\vartheta}$ and $\mathrm{E}_{\vartheta^{\prime}}$. Set

$$
\mathcal{E}=\bigcup_{\vartheta \in \Theta_{\Delta}(z)} \mathcal{E}_{\vartheta}
$$

That is, $\mathcal{E}$ includes all sets of edges $\mathrm{E}^{\prime} \subset \mathrm{E}_{\Delta}$ such that the corresponding graph $\mathrm{G}^{\prime}=\left(\Delta, \mathrm{E}^{\prime}\right)$ has at least one subgraph $\mathrm{G}_{\vartheta}, \vartheta \in \Theta_{\Delta}(z)$. Then (3.6) takes the form

$$
\begin{aligned}
M_{\Delta, z}^{\beta}(h \mid \xi) & -M_{\Delta, z}^{\beta}(h \mid \eta)=\frac{1}{\widetilde{Z}_{\Delta}^{\beta}(\xi) \widetilde{Z}_{\Delta}^{\beta}(\eta)} \\
& \times \sum_{\mathrm{E}^{\prime} \in \mathcal{E}} \int_{S^{\Delta}} \int_{S^{\Delta}}(h(\sigma(z))-h(\tilde{\sigma}(z))) \\
& \times \Gamma\left(\mathrm{E}^{\prime}\right) \Psi_{\Delta}(\xi, \eta) \chi_{\Delta}\left(d \sigma_{\Delta}\right) \chi_{\Delta}\left(d \tilde{\sigma}_{\Delta}\right) .
\end{aligned}
$$

In view of the positivity of all $\Gamma_{x y}$ and the fact that $h(\sigma) \in[0,1]$, the latter yields

$$
\begin{aligned}
\mid M_{\Delta, z}^{\beta}(h \mid \xi) & -M_{\Delta, z}^{\beta}(h \mid \eta) \mid \leq \frac{1}{\widetilde{Z}_{\Delta}^{\beta}(\xi) \widetilde{Z}_{\Delta}^{\beta}(\eta)} \\
& \times \sum_{E^{\prime} \in \mathcal{E}} \int_{S^{\Delta}} \int_{S^{\Delta}} \Gamma\left(\mathrm{E}^{\prime}\right) \Psi_{\Delta}(\xi, \eta) \chi_{\Delta}\left(d \sigma_{\Delta}\right) \chi_{\Delta}\left(d \tilde{\sigma}_{\Delta}\right) \\
& \leq \frac{1}{\widetilde{Z}_{\Delta}^{\beta}(\xi) \widetilde{Z}_{\Delta}^{\beta}(\eta)} \int_{S^{\Delta}} \int_{S^{\Delta}} \sum_{\vartheta \in \Theta_{\Delta}(z)} \Gamma\left(\mathrm{E}_{\vartheta}\right) \sum_{\mathrm{E}^{\prime} \in \mathcal{E}_{\vartheta}} \Gamma\left(\mathrm{E}^{\prime} \backslash \mathrm{E}_{\vartheta}\right) \\
& \times \Psi_{\Delta}(\xi, \eta) \chi_{\Delta}\left(d \sigma_{\Delta}\right) \chi_{\Delta}\left(d \tilde{\sigma}_{\Delta}\right) .
\end{aligned}
$$

To get the second inequality in (3.10) we used subadditivity like $\sum_{x \in A \cup B} \psi(x) \leq$ $\sum_{x \in A} \psi(x)+\sum_{x \in B} \psi(x), \psi(x) \geq 0$; which in our case is, see (3.9),

$$
\sum_{E^{\prime} \in \mathcal{E}} \leq \sum_{\vartheta \in \Theta_{\Delta}(z)} \sum_{E^{\prime} \in \mathcal{E}_{\vartheta}}
$$

as well as the fact that $\Gamma\left(\mathrm{E}^{\prime}\right)=\Gamma\left(\mathrm{E}_{\vartheta}\right) \Gamma\left(\mathrm{E}^{\prime} \backslash \mathrm{E}_{\vartheta}\right)$, see (3.7). Note that

$$
0 \leq \Gamma\left(\mathrm{E}_{\vartheta}\right) \leq \prod_{\langle x, y\rangle \in \mathrm{E}_{\vartheta}} \varkappa_{x y}(\beta),
$$


which follows by (3.8) and (3.1). We apply the latter upper bound in (3.10) and then obtain

$$
\begin{aligned}
\mid M_{\Delta, z}^{\beta}(h \mid \xi) & -M_{\Delta, z}^{\beta}(h \mid \eta) \mid \leq \frac{1}{\widetilde{Z}_{\Delta}^{\beta}(\xi) \widetilde{Z}_{\Delta}^{\beta}(\eta)} \sum_{\vartheta \in \Theta_{\Delta}(z)} \prod_{\langle x, y\rangle \in \mathrm{E}_{\vartheta}} \varkappa_{x y}(\beta) \\
& \times \int_{S^{\Delta}} \int_{S^{\Delta}} \prod_{\langle x, y\rangle \in \mathrm{E}_{\vartheta}}\left(1+\Gamma_{x y}\right) \\
& \left.\times \sum_{\mathrm{E}^{\prime \prime} \subset \mathrm{E}_{\Delta} \backslash \mathrm{E}_{\vartheta}} \Gamma\left(\mathrm{E}^{\prime \prime}\right) \Psi_{\Delta}(\xi, \eta)\right) \chi_{\Delta}\left(d \sigma_{\Delta}\right) \chi_{\Delta}\left(d \tilde{\sigma}_{\Delta}\right) \\
& =\sum_{\vartheta \in \Theta_{\Delta}(z)} \prod_{\langle x, y\rangle \in \mathrm{E}_{\vartheta}} \varkappa_{x y}(\beta),
\end{aligned}
$$

which by (3.2) yields (3.5). To get the latter equality in (3.11) we took into account that, see (3.6),

$$
\begin{aligned}
& \int_{S^{\Delta}} \int_{S^{\Delta}} \prod_{\langle x, y\rangle \in \mathrm{E}_{\vartheta}}\left(1+\Gamma_{x y}\right) \sum_{\mathrm{E}^{\prime \prime} \subset \mathrm{E}_{\Delta} \backslash \mathrm{E}_{\vartheta}} \Gamma\left(\mathrm{E}^{\prime \prime}\right) \\
& \times \Psi_{\Delta}(\xi, \eta) \chi_{\Delta}\left(d \sigma_{\Delta}\right) \chi_{\Delta}\left(d \tilde{\sigma}_{\Delta}\right) \\
& =\int_{S^{\Delta}} \int_{S^{\Delta}} \prod_{\langle x, y\rangle \in \mathrm{E}_{\Delta}}\left(1+\Gamma_{x y}\right) \\
& \times \Psi_{\Delta}(\xi, \eta) \chi_{\Delta}\left(d \sigma_{\Delta}\right) \chi_{\Delta}\left(d \tilde{\sigma}_{\Delta}\right)=\widetilde{Z}_{\Delta}^{\beta}(\xi) \widetilde{Z}_{\Delta}^{\beta}(\eta),
\end{aligned}
$$

which completes the proof.

Remark 1 A crucial aspect of the estimate in (3.5) is that it is uniform in the boundary spins $\xi$ and $\eta$. The main obstacle in proving uniqueness for Gibbs fields with $W_{x y}$ not satisfying item (i) of (2.12) is the lack of such uniformity.

Proof of Theorem 1 By [16, Theorem 1.33, page 23] the integrals as in (3.4) determine the specification $\left\{\pi_{\Delta}\right\}_{\Delta \Subset V}$; hence, the uniqueness in question will follow by

$$
\inf _{\Delta \Subset \mathrm{V}_{\xi, \eta \in S^{\vee}}} \sup _{\Delta, z} \mid M_{\Delta \mid \xi)-M_{\Delta, z}^{\beta}(h \mid \eta) \mid=0,}^{\beta}(h)
$$

holding (almost surely) for all $z \in \mathrm{V}$ and $h$ as in (3.4). To prove this, we fix $z$ and $h$, and then pick a cofinal sequence $\left\{\Delta_{l}\right\}_{l \in \mathbb{N}}$, for which we show that the sequence of random variable

$$
X_{l}^{\beta}=\sup _{\xi, \eta \in S^{\vee}}\left|M_{\Delta_{l}, z}^{\beta}(h \mid \xi)-M_{\Delta_{l}, z}^{\beta}(h \mid \eta)\right| \quad l \in \mathbb{N},
$$

converges to zero almost surely. Here cofinal means that: (a) $\Delta_{l} \subset \Delta_{l+1}, l \in \mathbb{N}$; (b) each $y \in \mathrm{V}$ lies in some $\Delta_{l}$. For the mentioned $z$, let $\left\{N_{k}\right\}_{k \in \mathbb{N}}$ be the sequence as in 
Definition 1. Then we set

$$
Y_{k}^{\beta}=\sup _{\xi, \eta \in S^{\vee}}\left|M_{\mathrm{V}_{N_{k}}, z}^{\beta}(h \mid \xi)-M_{\mathrm{V}_{N_{k}}, z}^{\beta}(h \mid \eta)\right| \quad k \in \mathbb{N}
$$

where $\mathrm{V}_{r}=\{x \in \mathrm{V}: \rho(z, x) \leq r\}$. By condition (ii) of (2.12) and (3.11) we get that

$$
\begin{aligned}
\mathbb{E}\left[Y_{k}^{\beta}\right] & \leq \sum_{\vartheta \in \Theta \mathrm{V}_{N_{k}}(z)} \mathbb{E}\left[\prod_{\langle x, y\rangle \in \mathrm{E}_{\vartheta}} \varkappa_{x y}(\beta)\right] \\
& =\sum_{\vartheta \in \Theta \mathrm{V}_{N_{k}}}(z) \prod_{\langle x, y\rangle \in \mathrm{E}_{\vartheta}} \mathbb{E}\left[\varkappa_{x y}(\beta)\right]=\sum_{\vartheta \in \Theta \mathrm{v}_{N_{k}}(z)} \varkappa^{\|\vartheta\|}(\beta),
\end{aligned}
$$

with $\varkappa(\beta):=\mathbb{E}\left[\varkappa_{x y}(\beta)\right]$. By condition (iii) of (2.12) and Lebesgue's dominated convergence theorem it follows that $\beta \mapsto \varkappa(\beta)$ is continuous and increasing, and $\varkappa(\beta) \rightarrow 0$ as $\beta \rightarrow 0$. Set $\beta_{*}$ such that $\varkappa\left(\beta_{*}\right)=e^{-\gamma}$, where $\gamma$ is as in (2.6), see also the first part of Proposition 4. Thus, $\varkappa(\beta) e^{\gamma}<1$ whenever $\beta<\beta_{*}$. Each $\vartheta \in \Theta \mathrm{V}_{N_{k}}(z)$ is a simple path of length at least $N_{k}$, and hence $\vartheta \in \Sigma_{N_{k}}^{N}(z)$ for some $N \geq N_{k}$. We take this into account in (3.14) and then obtain

$$
\mathbb{E}\left[Y_{k}^{\beta}\right] \leq \sum_{N=N_{k}}^{\infty} \sum_{\vartheta \in \Sigma_{N_{k}}^{N}(z)} \varkappa^{N}(\beta)=\sum_{N=N_{k}}^{\infty}\left|\Sigma_{N_{k}}^{N}(z)\right| \varkappa^{N}(\beta) \leq \frac{\left(\varkappa(\beta) e^{\gamma}\right)^{N_{k}}}{1-\varkappa(\beta) e^{\gamma}},
$$

where we used the estimate of $\left|\Sigma_{N_{k}}^{N}(z)\right|$ provided by Proposition 4 . Since $N_{k} \rightarrow+\infty$, by (3.15) we obtain that $Y_{k}^{\beta} \rightarrow 0$ in mean. Therefore, the sequence $\left\{Y_{k}^{\beta}\right\}_{k \in \mathbb{N}}$ contains a subsequence, $\left\{Y_{k_{l}}^{\beta}\right\}_{l \in \mathbb{N}}$, that converges to zero almost surely, see, e.g., [22, Theorem 3.4]. Then we set $\Delta_{l}=N_{k_{l}}$, and hence $X_{l}^{\beta}=Y_{k_{l}}^{\beta}$, see (3.12) and (3.13) and obtain the convergence to be proved.

Acknowledgements The authors thank both referees whose valuable and important remarks were essentially used in preparing the final version of the paper.

\section{Compliance with ethical standards}

Conflict of interest On behalf of all authors, the corresponding author states that there is no conflict of interest.

Open Access This article is licensed under a Creative Commons Attribution 4.0 International License, which permits use, sharing, adaptation, distribution and reproduction in any medium or format, as long as you give appropriate credit to the original author(s) and the source, provide a link to the Creative Commons licence, and indicate if changes were made. The images or other third party material in this article are included in the article's Creative Commons licence, unless indicated otherwise in a credit line to the material. If material is not included in the article's Creative Commons licence and your intended use is not permitted by statutory regulation or exceeds the permitted use, you will need to obtain permission directly from the copyright holder. To view a copy of this licence, visit http://creativecommons.org/licenses/by/4.0/. 


\section{References}

1. Kondratiev, Y., Kozitsky, Y., Pasurek, T.: Gibbs measures of disordered lattice systems with unbounded spins. Markov Process. Related Fields 18, 533-582 (2012)

2. von Dreifus, H., Klein, A., Perez, J.F.: Taming Griffiths' singularities: infinite differentiability of quenched correlation functions. Commun. Math. Phys. 170, 21-39 (1995)

3. Fröhlich, J., Imbrie, J.: Inproved perturbation expansion for disordered systems: beating Griffiths' singularities. Commun. Math. Phys. 96, 145-180 (1984)

4. Singh, R.R.P., Young, A.P.: Critical and Griffiths-McCoy singularities in quantum Ising spin glasses on $d$-dimensional hypercubic lattices: a series expansion study. Phys. Rev. E 96, 022139 (2017)

5. Bassalygo, L.A., Dobrushin, R.L.: Uniqueness of a Gibbs field with a random potential-an elementary approach. Theory Probab. Appl. 31, 572-589 (1986)

6. van den Berg, J.: A constructive mixing condition for 2-D Gibbs measures with random interactions. App. Probab. 25, 1316-1333 (1997)

7. Gielis, G., Maes, C.: The uniqueness regime of Gibbs fields with unbounded disorder. J. Stat. Phys. 81, 829-835 (1995)

8. Kȩpa, D., Kozitsky, Y.: Bassalygo-Dobrushin uniqueness for continuous spin systems on irregular graphs. Condens. Matter. Phys. 11, 313-329 (2008)

9. Kȩpa-Maksymowicz, D., Kozitsky, Y.: Paths and animals in infinite graphs with tempered degree growth. Discrete Appl. Math. 177, 137-145 (2014)

10. Kondratiev, Y., Kozitsky, Y., Pasurek, T.: Gibbs random fields with unbounded spins on unbounded degree graphs. J. Appl. Prob. 47, 856-875 (2010)

11. Häggström, O.: Markov random fields and percolation on general graphs. Ann. Appl. Probab. 32, 39-66 (2000)

12. de Angelis, G.F., de Falco, D., di Genova, G.: Random fields on Riemannian manifolds: a constructive approach. Commun. Math. Phys. 103, 297-303 (1986)

13. Burioni, R., Cassi, D.: Random walks on graphs: ideas, techniques and results. J. Phys. A Math. Gen. 38, R45-R78 (2005)

14. Daletskii, A., Kondratiev, Y., Kozitsky, Y.: Phase transitions in continuum ferromagnets with unbounded spins. J. Math. Phys. 56, 113502 (2015)

15. Romano, S., Zagrebnov, V.: Orientational ordering transition in a continuous-spin ferrofluid. Physica A 253, 483-497 (1998)

16. Georgii, H.-O.: Gibbs Measures and Phase Transitions. de Gruyter, New York (1988)

17. Dobrushin, R.L.: Perturbation methods of the theory of Gibbsian fields. In: Bernard, P. (ed.) Lectures on Probability Theory and Statistics. Lecture Notes in Mathematics, vol. 1648, pp. 1-66. Springer, Berlin (1994)

18. Cox, J.T., Gandolfi, A., Griffin, Ph.S., Kesten, H.: Greedy lattice animals I: Upper bounds. Ann. Appl. Probab. 3, 1151-1169 (1993)

19. Bondy, J.A., Murthy, U.S.R.: Graph Theory with Applications. The MacMillan Press Ltd., London (1976)

20. Clark, L., Gutman, I.: The exponent in the generalized Randić index. J. Math. Chem. 43, 32-44 (2008)

21. Kozitsky, Y.: On thermodynamic states of the Ising model on scale-free graphs. Condens. Matter Phys. 16, 1-12 (2013)

22. Gut, A.: Probability: A Graduate Course. Springer Texts in Statistics. Springer, New York (2005)

Publisher's Note Springer Nature remains neutral with regard to jurisdictional claims in published maps and institutional affiliations. 